\section{Sozialversicherungspflichtige Tätigkeiten - aktuelle Rechtsprechung zu Honorar- und Vertretungsärzten}

\section{Einführung}

Einer unserer früheren Beiträge behandelte bereits Rechtsentwicklungen in 2017 zur Einordnung der Tätigkeit eines Honorararztes hinsichtlich Arbeitnehmerstellung oder selbständiger Tätigkeit (RöFo 6/2017, S. 579-584).

Gemäß § 2 Abs. 1 S. 1 KHEntgG können Krankenhausleistungen - in den Grenzen des §20 Ärzte-ZV „auch durch nicht fest angestellte [...] Ärzte“" erbracht werden. Nach der Definition des BGH (BGH, MedR 2015, 120) ist unter einem Honorararzt ein Facharzt zu verstehen, der im stationären und/oder ambulanten Bereich des Krankenhauses ärztliche Leistungen für den Krankenhausträger erbringt, ohne bei diesem angestellt oder als Belegarzt oder Konsiliararzt tätig zu sein. Er wird zeitlich befristet freiberuflich auf Honorarbasis tätig, wobei das Honorar mit dem Krankenhausträger frei und unabhängig von den Vorgaben der Gebührenordnung für Ärzte (GOÄ) vereinbart wird.

Mit den Anforderungen der Rechtsprechung an die honorarärztliche Tätigkeit haben wir uns anhand eines Urteils des Landessozialgerichts (LSG) Baden-Württemberg (LSG BW, Urt. v. 21.2.2017, Az.: L 11 R 2433/16) ausführlich auseinandergesetzt. Mangels einer höchstrichterlichen Entscheidung, welche konkrete Maßstäbe zu der Statusbewertung von Honorarärzten aufstellt, bestanden erhebliche Unsicherheiten in Bezug auf die Bejahung der Selbstständigkeit von Honorarärzten.

In seiner Entscheidung hatte das LSG Baden-Württemberg darüber zu befinden, ob die Tätigkeit einer Radiologin als Praxisvertreterin im Rahmen eines versicherungspflichtigen Beschäftigungsverhältnisses ausgeübt wurde. Der Entscheidung waren Wertungen zu entnehmen, die sich auf Honorarärzte im Krankenhaus gleichsam übertragen ließen. Im konkreten Fall übernahm die Radiologin auf Grund mündlicher Vereinbarung Urlaubsvertretungen für die
Praxisinhaber einer radiologischen Gemeinschaftspraxis, die sie frei bestimmen konnte. Für ihre Tätigkeit stellte sie der Praxis Rechnungen nach einem vereinbarten Stundensatz. In Übereinstimmung mit der Vorinstanz bejahte das LSG Baden-Württemberg eine selbstständige Tätigkeit.

Der erkennende Senat des LSG stellte maßgeblich darauf ab, ob und inwieweit die Radiologin über das zwingend notwendige Maße hinaus in die Betriebsorganisation der Gemeinschaftspraxis eingegliedert war und einer nicht-fachlichen Weisungsgebundenheit unterlag. Im besprochenen Fall verneinte er im Rahmen einer Gesamtabwägung die Unselbstständigkeit ihrer Tätigkeit, da sie nicht in vergleichbarem Maße wie die angestellten Ärzte eingegliedert war. Ein wichtiges Indiz für das Vorliegen einer selbständigen Tätigkeit war, dass der Radiologin keine festen Arbeitszeiten vorgegeben oder Schichten ohne vorherige Absprache und gegen ihren Willen zugewiesen werden konnten. Vielmehr stand es ihr frei, an welchen Tagen sie eine Urlaubsvertretung übernehmen wollte. Das LSG Baden-Württemberg wies unter Berufung auf das BSG daraufhin, dass in Zweifelsfällen der tatsächliche Wille der Vertragsparteien maßgeblich ist, sofern dieser Wille den festgestellten sonstigen tatsächlichen Verhältnissen nicht offensichtlich widerspricht und er durch weitere Aspekte gestützt wird bzw. die übrigen Umstände gleichermaßen für Selbstständigkeit als auch für eine Beschäftigung sprechen.

Festzuhalten war, dass die Beurteilung der Selbstständigkeit - auch eines Honorararztes - durch eine Vielzahl von Einzelkriterien und Indizien geprägt ist und anhand einer Gesamtabwägung im Einzelfall festgestellt werden muss. Dabei galt generell, dass das Risiko der Verneinung einer selbstständigen Tätigkeit steigt, je mehr die Stellung des Honorararztes der eines angestellten Arztes gleicht.

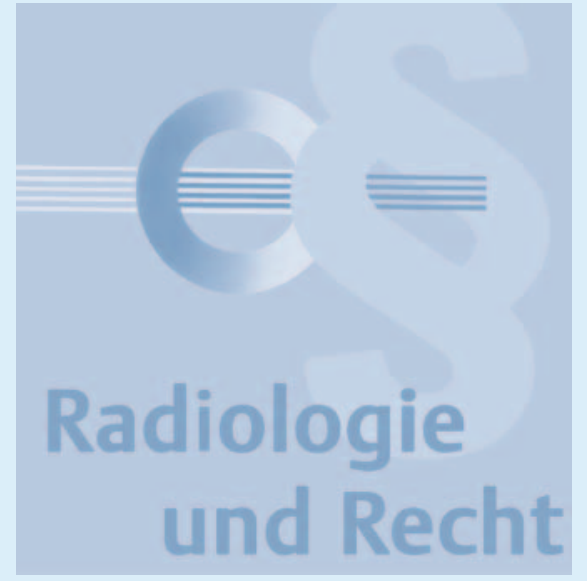

\section{Urteile des BSG in 2019}

Das Bundessozialgericht (BSG) hat im Anschluss mit diversen Urteilen vom 04.06.2019 (Az. B 12 R 11/18 R als Leitfall) zur Sozialversicherungspflicht der Tätigkeiten von Honorarärzten in Krankenhäusern für Aufregung gesorgt. Der erkennende Senat gelangte in den neun entschiedenen Revisionsverfahren zu dem Ergebnis, dass die Tätigkeit von Honorarärzten regelmäßig in einer abhängigen Beschäftigung ausgeübt wird und damit der Sozialversicherungspflicht unterliegt.

Die Landessozialgerichte haben in den zu entscheidenden Verfahren ganz überwiegend das Vorliegen einer (abhängigen) Beschäftigung und das Bestehen einer Versicherungspflicht in der Arbeitslosenversicherung und teilweise auch in der gesetzlichen Rentenversicherung (GRV) bejaht. Sie haben - mit unterschiedlicher Schwerpunktsetzung - auf die nach ständiger Rechtsprechung des entscheidenden Senats des BSG maßgeblichen Abgrenzungskriterien abgestellt und im Ergebnis bis auf in einem Ausnahmefall ein Überwiegen der Indizien für abhängige Beschäftigung (Weisungsgebundenheit und Eingliederung) angenommen. Die Tätigkeit prägende unternehmerische Risiken lägen nicht vor.

Die Ärzte und Krankenhäuser argumentierten unter anderem, dass Honorarärzte im Krankenhaus nach der Verkehrsanschauung sowie krankenhausvergütungsrechtlichen Regelungen als Selbstständige mit freiem Dienstvertrag anerkannt seien. Die Höhe der Vergütung spreche deutlich für eine 
selbstständige Tätigkeit. Die Nichtbehandlung eigener Patienten liege im Krankenhaus in der Natur der Sache und sei kein verwertbares Indiz. Da Krankenhausärzte in ihrer ärztlichen Tätigkeit eigenverantwortlich handelten, bestehe eine Weisungsbefugnis nicht; der Eingliederung komme insoweit keine eigenständige, jedenfalls aber keine entscheidende Bedeutung zu.

\section{Sachverhalt}

Dem Leitfall liegt folgender Sachverhalt zugrunde. Eine Fachärztin für Anästhesie wurde ab Januar 2013 auf Grundlage eines so bezeichneten „Konsiliararztvertrags“ auf Honorarbasis wiederholt im Tag- und Bereitschaftsdienst für einen Krankenhausbetreiber mit einem Stundenlohn von $80 €$ im Tagdienst und $64 €$ im Bereitschaftsdienst tätig. Sie war überwiegend im OP eingesetzt.

Im Oktober 2013 stellten der Krankenhausbetreiber und die Ärztin bei der DRV Bund den Antrag, für die Tätigkeit in den beiden betroffenen Krankenhäusern jeweils festzustellen, dass eine [abhängige] Beschäftigung nicht vorliege. Nach Anhörung stellte die DRV Bund fest, dass die Ärztin in beiden Kliniken im Rahmen eines abhängigen Beschäftigungsverhältnisses tätig sei. Es bestehe Versicherungspflicht in der GRV und nach dem Recht der Arbeitsförderung.

Das Sozialgericht (SG) stellte auf die Klage hiergegen fest, dass die Ärztin in beiden Krankenhäusern selbstständig tätig und nicht abhängig beschäftigt sei und nicht der Versicherungspflicht unterliege. Das LSG hat das Urteil des SG aufgehoben und die Klagen abgewiesen.

\section{Begründung}

Das BSG stellte fest, dass die Ärztin in ihrer Tätigkeit als Anästhesistin in den Krankenhäusern gegen Arbeitsentgelt abhängig beschäftigt und deshalb nach dem Recht der Arbeitsförderung versicherungspflichtig war.

Nach der ständigen Rechtsprechung des BSG setzt eine abhängige Beschäftigung voraus, dass der Arbeitnehmer vom Arbeitgeber persönlich abhängig ist. Bei einer Beschäftigung in einem fremden Betrieb ist dies der Fall, wenn der Beschäftigte in den
Betrieb eingegliedert ist und dabei einem Zeit, Dauer, Ort und Art der Ausführung umfassenden Weisungsrecht des Arbeitgebers unterliegt. Diese Weisungsgebundenheit kann eingeschränkt und zur „funktionsgerecht dienenden Teilhabe am Arbeitsprozess" verfeinert sein. Demgegenüber ist eine selbstständige Tätigkeit vornehmlich durch das eigene Unternehmerrisiko, das Vorhandensein einer eigenen Betriebsstätte, die Verfügungsmöglichkeit über die eigene Arbeitskraft und die im Wesentlichen frei gestaltete Tätigkeit und Arbeitszeit gekennzeichnet.

Ob jemand beschäftigt oder selbstständig tätig ist, richtet sich danach, welche Umstände das Gesamtbild der Arbeitsleistung prägen und hängt davon ab, welche Merkmale überwiegen. Die Zuordnung setzt voraus, dass alle nach Lage des Einzelfalls als Indizien in Betracht kommenden Umstände festgestellt, in ihrer Tragweite zutreffend erkannt und gewichtet, in die Gesamtschau mit diesem Gewicht eingestellt und nachvollziehbar, also den Gesetzen der Logik entsprechend und widerspruchsfrei gegeneinander abgewogen werden.

Bei der Statusbeurteilung ist regelmäßig vom Inhalt sämtlicher zwischen den Beteiligten getroffenen Vereinbarungen auszugehen, unabhängig davon ob schriftlich, mündlich oder stillschweigend vereinbart. Erst auf der Grundlage dieser Feststellungen über den (wahren) Inhalt der Vereinbarungen ist eine wertende Zuordnung des Rechtsverhältnisses zum Typus der Beschäftigung oder selbstständigen Tätigkeit vorzunehmen und in einem weiteren Schritt zu prüfen, ob besondere Umstände vorliegen, die eine hiervon abweichende Beurteilung notwendig machen.

Für die Beurteilung der Tätigkeit von Honorarärzten gelten keine abweichenden Maßstäbe. Eine bloße Bezeichnung als „Honorararzt“ kennzeichnet sozialversicherungsrechtlich kein besonderes Tätigkeitsbild. Es spielt keine entscheidende Rolle, ob nach der Verkehrsanschauung anerkannt ist, dass so bezeichnete Honorarärzte im Krankenhaus selbstständig tätig sind oder zumindest sein können. Auch auf die Einordnung von Honorarverträgen durch die Arbeitsgerichte kommt es nicht an, da ein vollständiger Gleichklang zwischen dem Arbeitnehmer- und dem Beschäftigtenbegriff nach $\S 7$ Abs. 1 SGB IV nicht besteht.

Das vorinstanzliche LSG hat ausgehend von den Maßstäben zur Beurteilung des Vorliegens von Beschäftigung eine zutreffende Gesamtwürdigung der jeweiligen Einzeleinsätze vorgenommen.

Übernahm die Ärztin Dienste, wurde sie als Vertreterin eines angestellten Arztes eingesetzt und verrichtete die gleichen Arbeiten. Ein Dienstzimmer wurde ihr bei Bereitschaftsdiensten kostenlos bereitgestellt; dies war auch bei beschäftigten Ärzten üblich. Sie wurde ausschließlich in den Räumen der Kliniken, überwiegend in Operationssälen, tätig und nutzte die dortigen Betriebsmittel. Sie war im Dienstplan der Kliniken eingetragen und arbeitete arbeitsteilig mit anderen Mitarbeitern zusammen. Nach außen war sie nicht als externe Mitarbeiterin erkennbar. Sie behandelte ausschließlich Patienten der Kliniken. In fachlicher Hinsicht war die Ärztin grundsätzlich eigenverantwortlich und weisungsfrei tätig. Ausgehend von diesen Feststellungen ist das LSG zu Recht zu dem Ergebnis gekommen, dass die Indizien für eine abhängige Beschäftigung der Ärztin überwiegen.

\section{Aktuelle Urteile}

Am 19.10.2021 hatte das BSG erneut in Angelegenheiten des Versicherungs- und Beitragsrechts über sechs Revisionen zu entscheiden. Bis zum Ende der Erstellung dieses Beitrages waren die vollständigen Entscheidungen noch nicht veröffentlicht. Aus dem Terminbericht des genannten Datums ergeben sich vorab die zusammengefassten Ergebnisse.

Von besonderem Interesse erscheint das Urteil hinsichtlich einer Vertretungstätigkeit, Az. B 12 R 1/21 R. Das BSG stufte die ärztliche Vertretungstätigkeit in einer Gemeinschaftspraxis als abhängige Beschäftigung ein. Die Ärztin war insbesondere hinsichtlich der Zuweisung bestimmter Patienten weisungsgebunden. Aufgrund des arbeitsteiligen Zusammenwirkens mit dem Praxispersonal und der kostenfreien Nutzung von Einrichtungen und Mitteln 
der Gemeinschaftspraxis war sie in deren Arbeitsabläufe eingegliedert. Das ausschließliche Tätigwerden in einer Vertretungssituation änderte daran nichts. Der Eingliederung in einen fremden „Arztbetrieb“ könne es zwar entgegenstehen, wenn ein Arztvertreter für die Dauer seiner Tätigkeit die Stelle des Praxisinhabers einnimmt und zeitweilig selbst dessen Arbeitgeberfunktionen erfüllt. Das sei hier aber nicht der Fall. Die Ärztin habe lediglich die ärztlichen Leistungen vertretungsweise erbracht und keine Vertretung in der Rechtsstellung der Mitglieder der Gemeinschaftspraxis geleistet. Ob mit der gewählten Ausgestaltung der ärztlichen Vertretung berufszulassungsrechtlichen Anforderungen Genüge getan werde, sei für die sozialversicherungsrechtliche Einordnung einer Tätigkeit als Beschäftigung unerheblich.

Im Übrigen stellten die Entscheidungen insbesondere eine abhängige Beschäftigung und damit Sozialversicherungspflicht von Notärzten im Rettungsdienst, aber auch von ambulanten Altenpflegerinnen in der Intensivpflege bei einem Pflegedienst fest.

\section{Folgen der Entscheidungen}

Die jüngsten Urteile scheinen die bisherige Beurteilung fortzuschreiben und zu verschärfen. Maßgeblich war und ist weiterhin eine umfassende Abwägung sämtlicher Umstände im Einzelfall. In Anbetracht der zunehmenden Entscheidungen in Einzelfällen, in denen eine Sozialversicherungspflicht für unterschiedliche Tätigkeiten festgestellt wird, besteht derzeit offenbar eine klare Tendenz zur Bejahung einer Sozialversicherungspflicht; unabhängig ob als Honorar-, Vertretungs- oder Notarzt. Insbesondere im Hinblick auf die aktuellsten Urteile erscheint fraglich, inwieweit noch das eingangs behandelte Urteil aus 2017 des LSG Baden-Württemberg zur Vertretungstätigkeit heute hätte bestehen bleiben können.

In der Folge erscheinen nahezu sämtliche ärztliche Tätigkeiten, die nicht als abhängiges Beschäftigungsverhältnis ausgestaltet sind und Verbindungen zu Tätigkeiten von Angestellten aufweisen, als risikobehaftet. Dies dürfte dazu führen, dass nunmehr auch mit Vertretungsärzten in der Regel Anstellungsverträge abgeschlossen werden sollten. Zu berücksichtigen ist hierbei, dass in der Praxis der Vertretungsbedarf weit überwiegend lediglich vorübergehend sein wird und demnach nur befristete Verträge in Betracht kommen. In diesem Zusammenhang kann zumindest helfen, dass die Beschäftigung zur Vertretung eines anderen Arbeitnehmers einen sachlichen Grund für eine Befristung nach $\S 14$ Abs. 1 Satz 2 Nr. 3 Teilzeit- und Befristungsgesetz (TzBfG) darstellt. Wenn ein derartiger Sachgrund tatsächlich vorliegt, ist zudem unschädlich, wenn zuvor bereits ein ohne Sachgrund befristeter Vertrag mit demselben Arbeitnehmer bestand.

Prof. Dr. Peter Wigge

Rechtsanwalt

Fachanwalt für Medizinrecht

Tilmann Kirsch

Rechtsanwalt

Fachanwalt für Medizinrecht

Rechtsanwälte Wigge

Großer Burstah 42

20457 Hamburg

Telefon: (040) 3398 705-90

Telefax: (040) 3398 705-99

Internet: www.ra-wigge.de

E-Mail: kanzlei@ra-wigge.de 